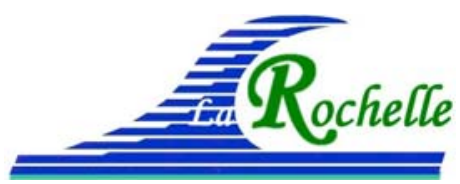

XVèmes Journées Nationales Génie Côtier - Génie Civil

La Rochelle, 29 au 31 mai 2018

DOI:10.5150/jngcgc.2018.029

(c) Editions Paralia CFL

disponible en ligne - $h$ ttp://www.paralia.fr - available online

\title{
Migration de la barre de déferlement par effet des ondes longues
}

\author{
Hervé MiCHALleT ${ }^{1}$, Mariana V.L. ROCHA ${ }^{1,2}$, Paulo A. SILVA ${ }^{2}$
}

1. Laboratoire des Ecoulements Géophysiques et Industriels, UMR 5519 CNRS, Université Grenoble-Alpes, Grenoble-INP, CS 40700, 38058 Grenoble Cedex 9 herve.michallet@legi.cnrs.fr

2. CESAM \& Department of Physics, Univ. Aveiro, Portugal. marianavlmrocha@gmail.com; psilva@ua.pt

\section{Résumé :}

Des expériences sont conçues pour reproduire la morphodynamique cross-shore de barres d'avant-côte dans un modèle réduit de plage constituée de sédiment léger. Des paquets d'ondes bichromatiques avec leurs ondes longues (infragravitaires) liées, et des ondes longues libres dans certains cas, se propagent et forment le profil de plage. Les hauteurs de vague des ondes courtes sont identiques pour toutes les conditions, tandis que les ondes longues diffèrent en amplitude et en phase. La non-linéarité des vagues (quantifiée par les asymétries horizontale et verticale) est similaire avant et sur la barre de déferlement. Un transport sédimentaire vers le large et une migration de la barre sont observés pour les conditions présentant une forte composante infragravitaire. Des mesures vidéo montrent qu'une suspension dense en sédiment, produit par le déferlement des plus grandes vagues, est ensuite advectée vers le large par l'écoulement associé aux ondes longues.

\section{Mots-clés :}

Génie côtier, Environnement littoral, Transport sédimentaire, Morphodynamique de plage, Asymétries de vague, PIV.

\section{Introduction}

Les ondes infragravitaires (IG) sont des ondes longues de période de l'ordre de 25 à 250 $\mathrm{s}$, généralement produites par l'interaction des différentes composantes fréquentielles d'un état de mer formé par le vent. On parle alors d'ondes liées, puisqu'elles se propagent à la vitesse de groupe des ondes courtes. Elles sont connues pour avoir un impact important sur la morphologie des plages, voir BERTIN et al. (2018).

Il est ainsi communément admis qu'elles peuvent contribuer notablement à l'érosion du haut de plage et au transport sédimentaire vers le large en zone de déferlement. Ceci reste toutefois difficile à quantifier en nature devant les autres effets (influence du courant moyen ou undertow, vagues fortement asymétriques, etc.). Les modèles numériques de morphodynamique ne parviennent pas à très bien reproduire les migrations des barres d'avant-côte, une des raisons peut être que l'effet des ondes IG 


\section{Thème 2 - Dynamique sédimentaire}

n'est généralement par pris en compte dans ces modèles (voir par exemple DUBARBIER et al. 2015).

ROELVINK \& STIVE (1989) ont montré par des expériences de laboratoire que les ondes longues contribuent à la migration vers le large de la barre de déferlement. On peut reprocher à ces expériences en modèle réduit d'amplifier le transport de sédiment en suspension devant le transport par charriage. Utilisant du sable, la réduction d'échelle ne permet pas de vérifier simultanément les nombres de Rouse et de Shields caractérisant respectivement le transport en suspension et par charriage. Pour pallier à ce défaut, GRASSO et al. (2009) ont utilisé un sédiment plastique de faible densité et montré que des morphodynamiques de plage pouvaient être reproduites en laboratoire.

Nous décrivons ici un protocole pour isoler l'effet des ondes IG sur la dynamique de la barre de déferlement (section 2). Nous mettons en évidence des migrations de barre vers le large ou vers la côte, en fonction de la composante basse fréquence de la houle incidente à la plage (section3). Des mesures vidéo permettent d'évaluer le transport sédimentaire associé aux ondes longues (section 4).

\section{Dispositif expérimental}

Les expériences sont décrites en détail par ROCHA (2016). Un canal à houle de $36 \mathrm{~m}$ de long et $0.55 \mathrm{~m}$ de large est rempli de sédiment plastique (PMMA, densité 1.185, diamètre médian $0.65 \mathrm{~mm}$ ). Des paquets d'ondes bichromatiques sont produits par un batteur piston situé à l'une de ses extrémités, se propagent et modèlent la plage. L'élévation de la surface libre est mesurée en 12 positions le long du profil de plage par des sondes capacitives. Le profil de plage est mesuré par une sonde optique, montée sur un charriot motorisé, qui émet un faisceau laser impactant le lit de sédiment.

Le choix de paquets d'ondes bichromatiques permet de conserver les mêmes caractéristiques de vagues (hauteur, période) et de ne modifier que les ondes IG (en terme d'amplitude et de phase vis-à-vis de l'enveloppe des vagues).

\section{Mise en évidence de migrations de barres}

Des exemples d'élévation de la surface libre à l'approche de la plage sont montrés figure 1a, pour lesquelles la condition $\mathrm{C} 2$ est un paquet de vagues constitué de 2 groupes avec une modulation maximum $\left(a_{1}=a_{2}=0.03 \mathrm{~m}, f_{1}=0.5515 \mathrm{~Hz}\right.$ et $f_{2}=0.6250$ $\mathrm{Hz})$ tandis que C3 présente une modulation moindre $\left(a_{1}=0.0413 \mathrm{~m}\right.$ et $a_{2}=0.01 \mathrm{~m}$, et mêmes fréquences $f_{1}$ et $f_{2}$ ) et donc une onde longue liée de plus faible amplitude.

Dans cette expérience, le paquet de vagues C2 est reproduit trois fois, en attendant le repos entre chaque essai. Le profil de plage est mesuré avant de réaliser trois fois la condition $\mathrm{C} 3$. 


\section{XVèmes Journées Nationales Génie Côtier - Génie Civil \\ La Rochelle, 29 au 31 mai 2018}
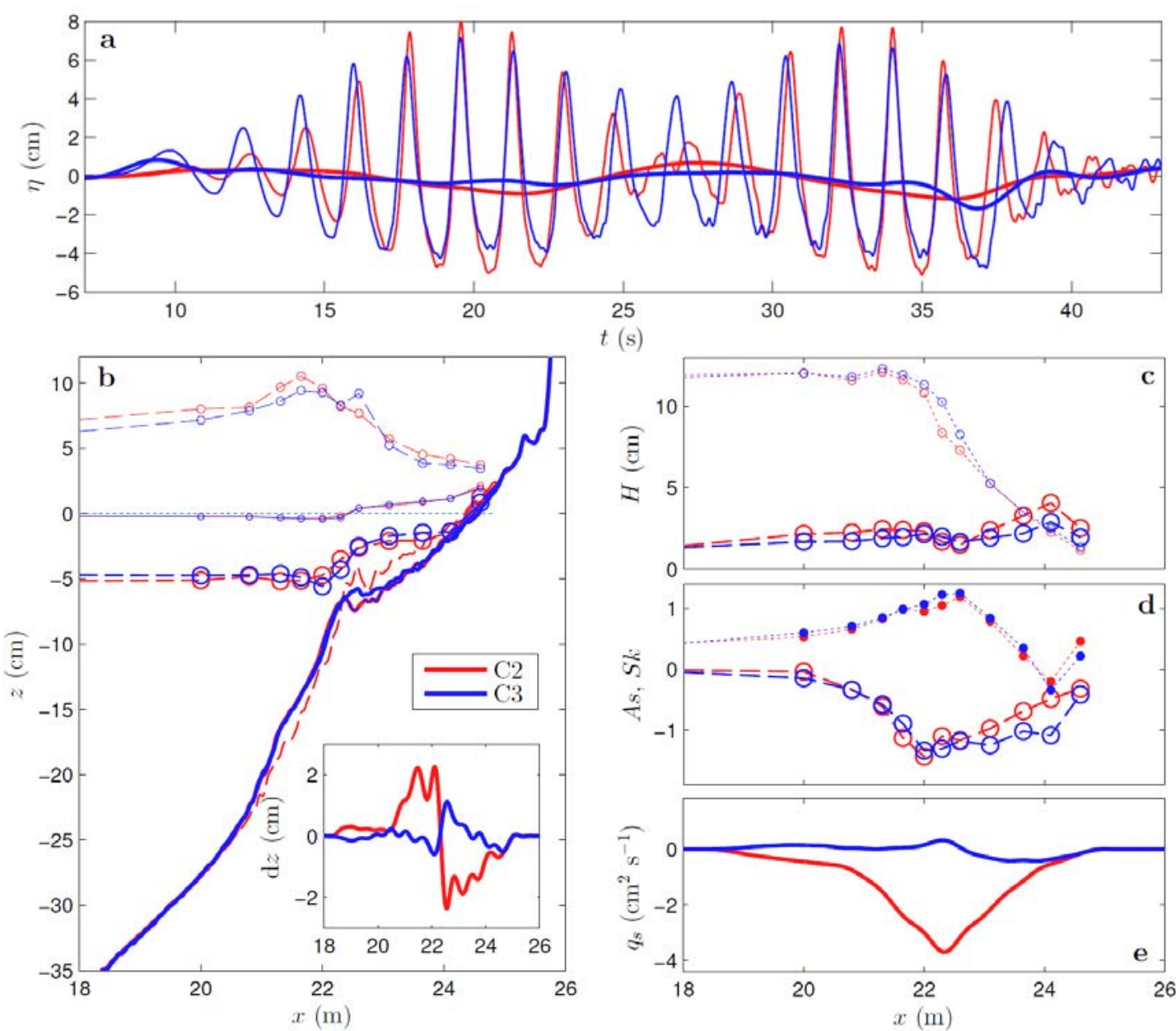

Figure 1. a: Série temporelle des déplacements de surface libre (traits fins) et composantes infragravitaires (traits gras) en $x=18.5 \mathrm{~m}$ ( $x=0$ est la position moyenne $d u$ batteur) pour les conditions C2 (en rouge) et C3 (en bleu). $b$ : Profils de plages (finaux en traits gras, les tirets rouges représentent le profil initial pour la condition C2) résultant de 3 essais C2 (rouge) puis 3 essais C3 (bleu), avec les niveaux d'eau maximum (cercles), moyen (petits symboles) et minimum (grands cercles); variations de fond en insert. $c$ : Hauteur de vague des ondes courtes (petits cercles) et des ondes longues (grands cercles). $d$ : Asymétries verticale (Sk, petits symboles) et horizontale (As, grands cercles). e : Transport sédimentaire.

Les profils de plage sont marqués par une barre de déferlement en $x=22 \mathrm{~m}$ sur laquelle les vagues se brisent (figure 1b), ainsi que le montre la décroissance brutale du maximum d'élévation de surface libre.

Les statistiques pour les deux trains d'ondes sont très similaires, en terme de variation cross-shore de hauteur de vague des ondes courtes (petits symboles figure 1c), de paramètres non-linéaires (figure 1d) que sont le wave skewness 


\section{Thème 2 - Dynamique sédimentaire}

$$
S k=\frac{\left\langle(\eta-\bar{\eta})^{3}\right\rangle}{\sigma_{\eta}^{3}}
$$

et le wave asymmetry

$$
\text { As }=\frac{\left\langle\mathscr{H}(\eta-\bar{\eta})^{3}\right\rangle}{\sigma_{\eta}^{3}}
$$

où $\eta$ est l'élévation de la surface libre, de niveau moyen $\bar{\eta}$, d'écart type $\sigma_{\eta},<>$ représente la moyenne temporelle sur la durée totale du paquet de vagues $2 /\left(f_{2}-f_{1}\right)=$ $27.2 \mathrm{~s}$, et $\mathscr{H}$ est la partie imaginaire de la transformée de Hilbert.

Statistiquement, la seule différence notable entre les deux conditions est l'amplitude de la composante basse fréquence (grands cercles figure 1c), environ $30 \%$ plus grande pour $\mathrm{C} 2$ que pour $\mathrm{C} 3$ à l'approche de la plage. On notera un minimum local juste après le point de déferlement (en $x \approx 22.7 \mathrm{~m}$ ) caractéristique d'une structure de type onde stationnaire et donc d'une réflexion partielle de l'onde longue. Cela se traduit nécessairement par un maximum de la composante basse fréquence de vitesse horizontale dans cette zone.

Les évolutions morphologiques permettent de calculer le transport sédimentaire

$$
q_{s}(x)=-(1-p) \int_{x_{i}}^{x} \Delta h_{s}(x) d x
$$

où $\Delta h_{s}$ est la différence de niveau du lit sédimentaire entre deux profils, $x_{i}$ la position cross-shore correspondant à la profondeur de coupure (pour laquelle $\Delta h_{s}=0$ ) et $p \approx 0.5$ est la porosité du lit, qui peut varier légèrement au cours d'un essai, et peut donc être ajustée (selon la procédure décrite par GRASSO, 2009) pour vérifier $q_{s}\left(x_{f}\right)=0$, avec $x_{f}=$ $29 \mathrm{~m}$ une position sur la dune qui ne change pas d'un essai à l'autre.

Les transports sédimentaires pour les conditions C2 et C3 sont comparés sur la figure 1e. Tandis que le paquet de vagues $\mathrm{C} 2$ conduit clairement à une migration de la barre de déferlement vers le large, la condition $\mathrm{C} 3$ produit un léger transport vers la côte. Le profil final (en bleu gras figure 1a) est en fait très proche du profil de plage en équilibre pour la condition $\mathrm{C} 3$.

Les statistiques de vagues et l'évolution du profil de plage de la condition C2 sont comparées sur la figure 2 aux résultats d'une condition $\mathrm{C} 1$, pour laquelle une onde longue libre a été ajoutée. La figure 2a montre que la composante basse fréquence du signal d'élévation de la surface libre à l'approche de la plage est amplifiée pour C1 par rapport à $\mathrm{C} 2$. Pour cette expérience, le paquet de vagues $\mathrm{C} 1$ est envoyé sur la plage, puis $\mathrm{C} 2$, et de nouveau $\mathrm{C} 1$ puis $\mathrm{C} 2$. On attend le repos pour réaliser la mesure du profil de plage entre chaque paquet. Les statistiques de vagues sont très similaires pour les deux conditions, en terme de maximum et minimum de $\eta$ (figure 2b), de $S k$ et As (figure 2d), de hauteur de vague des ondes courtes (petits symboles figure $2 \mathrm{c}$ ), surtout jusqu'à la zone de déferlement $(x<23 \mathrm{~m})$. La différence notable est la hauteur de vague de la composante IG (grands symboles figure 2c). Le transport sédimentaire résultant (figure 


\section{XVèmes Journées Nationales Génie Côtier - Génie Civil La Rochelle, 29 au 31 mai 2018}

2e) est dirigé vers la côte pour la condition $\mathrm{C} 2$, et vers le large pour la condition $\mathrm{C}$ 1. Nous mettons ici en évidence que, pour un profil de plage proche de l'équilibre pour ce qui est du transport induit par les ondes courtes, une amplification de la composante IG favorise la migration de la barre de déferlement vers le large.

Une augmentation de l'amplitude des ondes IG, et de la vitesse associée, ne suffisent pas à expliquer cette migration. Les ondes IG, longues et de relativement faibles amplitudes, sont censées être quasi-symétriques et donc transporter autant vers la côte que vers le large. Des mesures vidéo ont été réalisées pour mieux comprendre le phénomène.
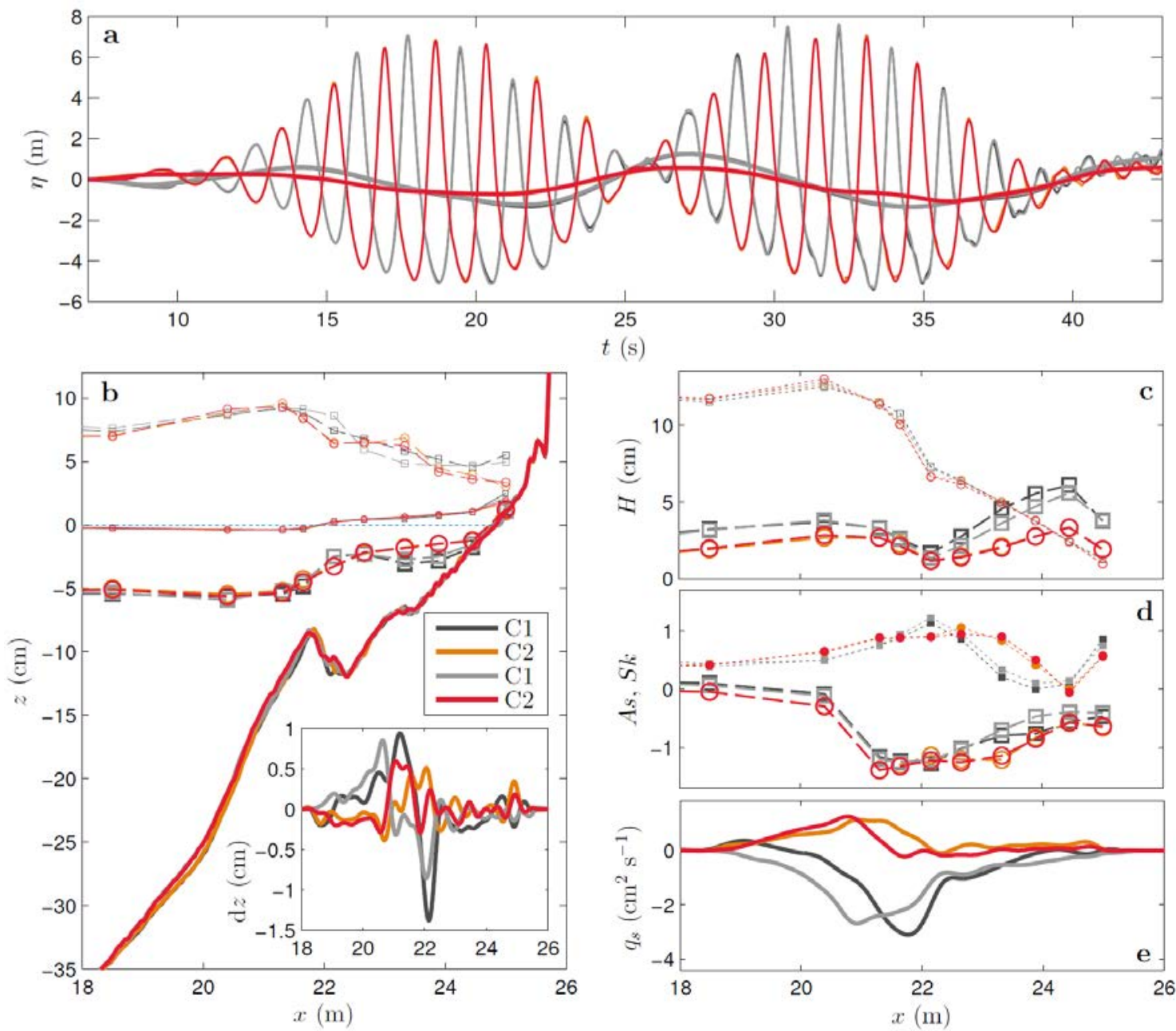

Figure 2. Idem Figure 1, pour 4 essais successifs : C1 (en noir), C2 (orange), C1 (gris), C2 (rouge).

\section{Observations du déferlement et du transport sédimentaire par vidéo}

Des vidéos ont été réalisées à travers la paroi latérale en verre du canal avec une caméra rapide (200 Hz, 1280 x 800 pixels). Deux exemples d'images extraites d'une vidéo pour 


\section{Thème 2 - Dynamique sédimentaire}

la condition $\mathrm{C} 2$ sont montrés sur la figure 3. Les images sont traitées pour suivre la surface libre, identifier la présence des bulles d'air entraînées par le déferlement, quantifier la présence de sédiment dans l'écoulement (par analyse des niveaux de gris), et obtenir les champs de vitesse par corrélation entre images successives (Correlation Image Velocimetry).
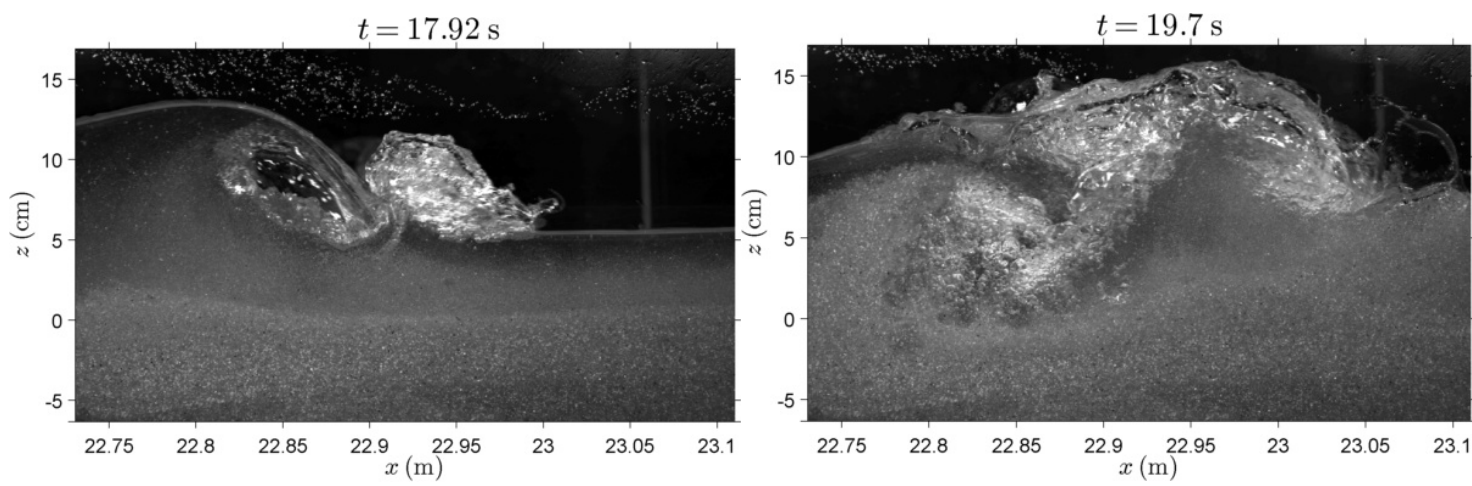

Figure 3. Illustrations du déferlement et de la mobilisation du sédiment pour 2 vagues de la condition C2. Le sommet de la barre de déferlement se situe en $x \approx 22.7 \mathrm{~m}$.

Un exemple de séries temporelles de la vitesse horizontale et de la concentration est montré figure 4, pour une position légèrement en amont de la barre de déferlement. Le niveau du lit est en $z=0$ avant l'arrivée du paquet. Au cours de l'essai, on peut considérer le lit comme étant la zone de vitesse nulle et de concentration $c>0.4$. Sous les fronts de vague, le substrat sédimentaire peut être mobilisé brièvement sur une profondeur de plusieurs centimètres (par exemple à $t=5 \mathrm{~s}$, figure 4), révélant un transport de type plug flow (voir BERNI et al. 2017). A la vague suivante ( $t=6.7 \mathrm{~s}$ ), la couche de sédiment est mise en suspension de sorte qu'elle est transportée vers le large sous les creux des vagues suivantes. Cela se traduit par une profondeur d'érosion pouvant atteindre environ $4 \mathrm{~cm}$ par rapport à la situation au repos. Le sédiment ne se redépose complètement qu'à la fin du paquet $(t \approx 35 \mathrm{~s})$.

Ces mesures illustrent qu'il faut plusieurs vagues pour déstabiliser en profondeur le substrat sédimentaire, et que la quantité de sédiment en suspension offerte au transport est plus importante dans la deuxième moitié de chaque groupe de vagues.

Les flux sédimentaires présentés sur la figure 5 mettent en évidence que le transport vers le large se produit entre le milieu et la fin de chaque groupe de vagues, conditionné par la composante basse fréquence. Le maximum de vitesse IG vers le large se produit au moment où la concentration en sédiment est la plus forte dans toute la colonne d'eau ( $t=10-12 \mathrm{~s}$ et $t=22-24 \mathrm{~s}$ ). Les profils verticaux de vitesse moyenne, de concentration et de flux (bas de la figure 5) montrent une bonne reproductibilité pour chaque groupe du paquet. On notera que l'essentiel du flux sédimentaire se produit sous le niveau du lit au repos (pour $z<0$ ). 


\section{XVèmes Journées Nationales Génie Côtier - Génie Civil La Rochelle, 29 au 31 mai 2018}
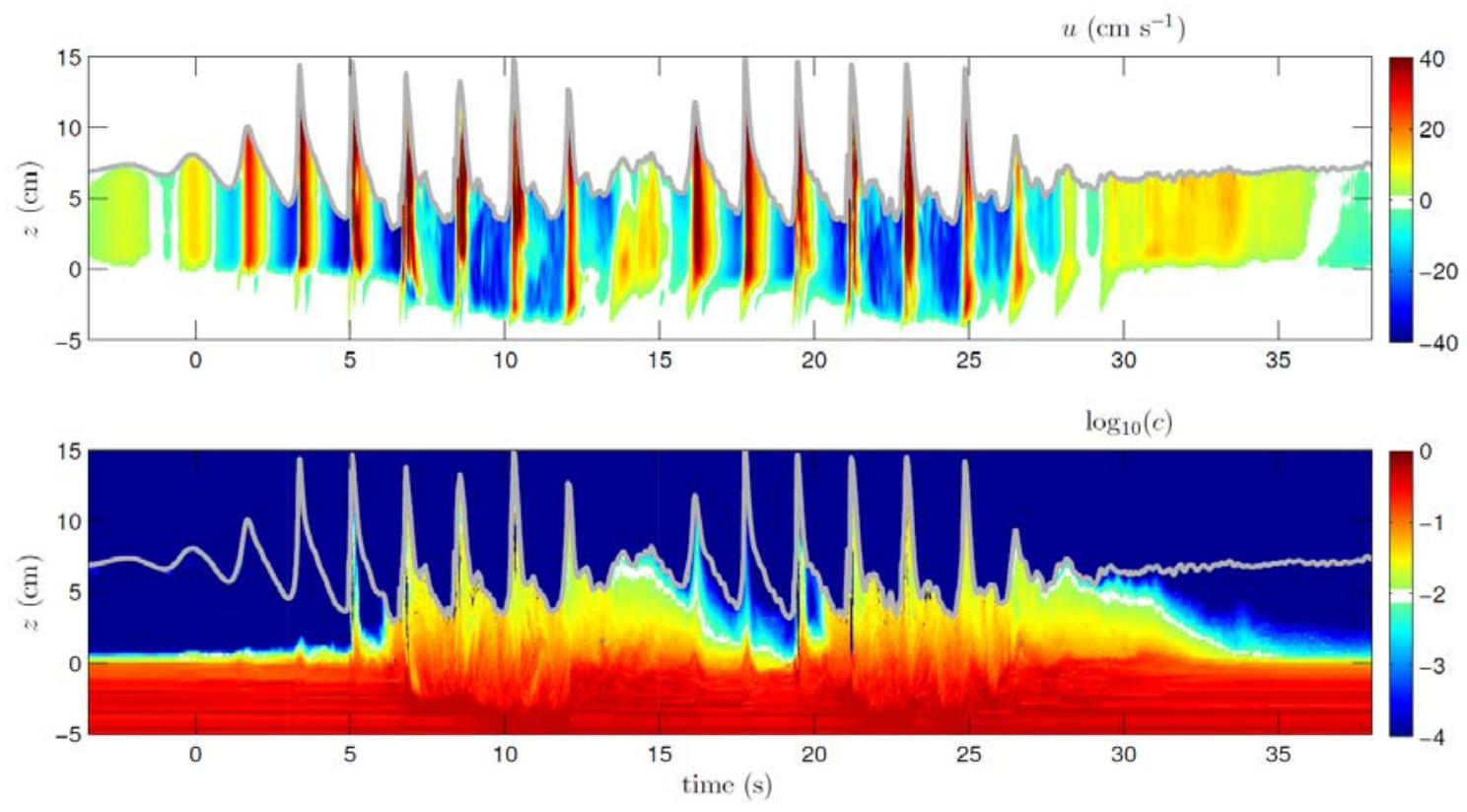

Figure 4. Haut : série temporelle de la vitesse horizontale en $x=22.74 \mathrm{~m}$ pour C2. Bas : concentration en sédiment correspondante. Le trait gris représente l'élévation de la surface libre.

\section{Conclusions}

Des expériences de laboratoire en modèle réduit ont été réalisées pour mettre en évidence le transport sédimentaire associé aux ondes IG. Pour un profil de plage proche de l'équilibre, une augmentation de l'énergie des ondes IG favorise la migration de la barre de déferlement vers le large. Ceci s'explique du fait que, sur la barre, le maximum de vitesse IG vers le large se produit dans la deuxième moitié de chaque groupe de vague, lorsque l'érosion induite par le déferlement des vagues est maximum et la suspension dans la colonne d'eau est la plus intense.

\section{Références bibliographiques}

BERNI C., MICHALLET H., BARTHELEMY E. (2017). Effects of horizontal pressure gradients on bed destabilization under waves. J. Fluid Mech., Vol. 812, pp 721-751. https://doi.org/10.1017/jfm.2016.805

BERTIN X. et al. (2018). Infragravity waves: From driving mechanisms to impacts. Earth-Science Reviews, Vol. 177, pp 774-799. doi: 10.1016/j.earscirev.2018.01.002.

DUBARBIER B., CASTELLE B., MARIEU V., RUESSINK G. (2015). Process-based modeling of cross-shore sandbar behavior. Coastal Engineering, Vol. 95, pp 35-50. https://doi.org/10.1016/j.coastaleng.2014.09.004

GRASSO F., MICHALLET H., BARTHELEMY E., CERTAIN R. (2009). Physical modeling of intermediate cross-shore beach morphology: Transients and equilibrium states. J. Geophys. Res., Vol. 114, C09001. https://doi.org/10.1029/2009JC005308 


\section{Thème 2 - Dynamique sédimentaire}
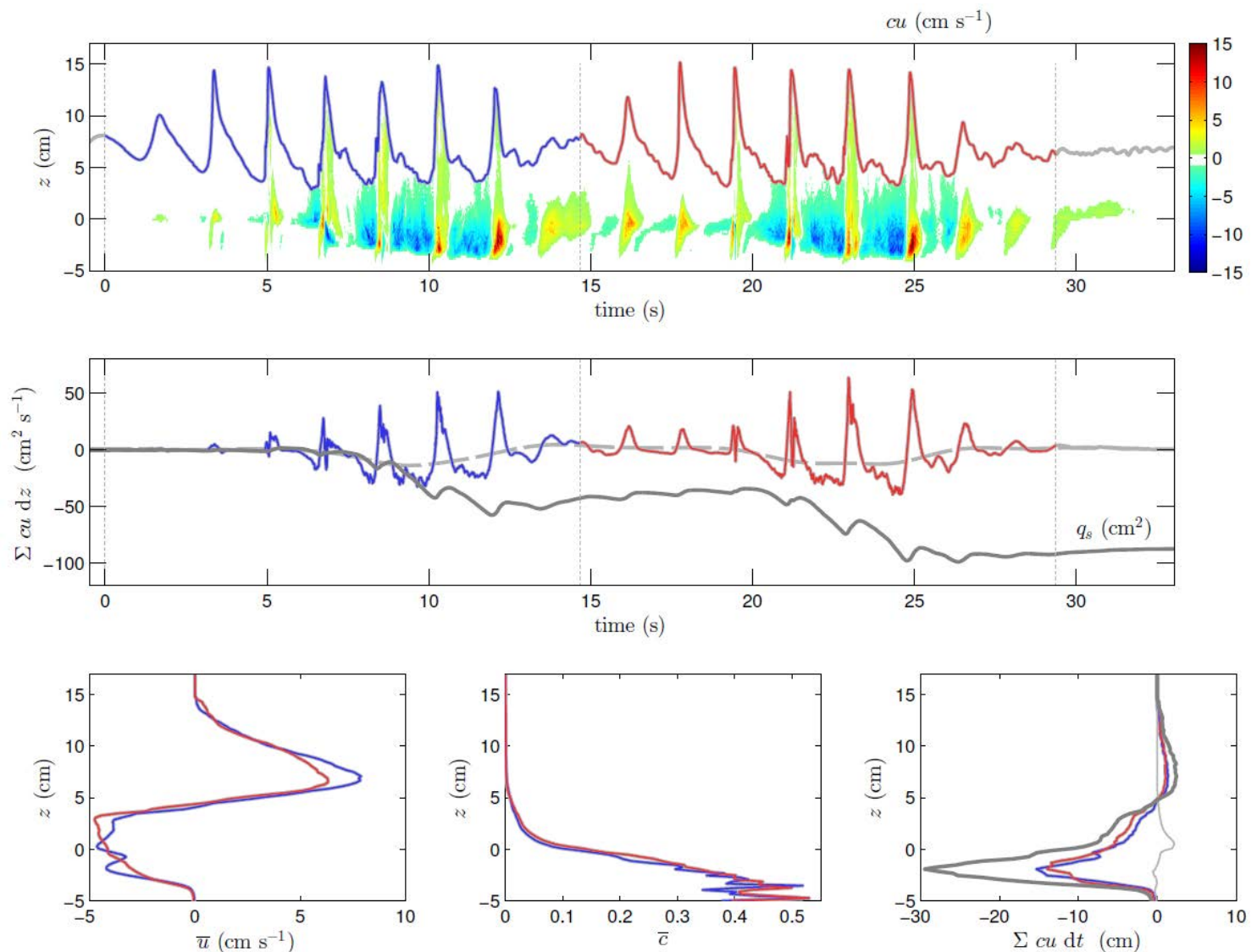

Figure 5. Haut : série temporelle du flux sédimentaire sur la verticale (la courbe représente l'élévation de la surface libre), les lignes pointillées verticales séparent les deux groupes de vagues. Milieu : flux intégré sur la verticale (traits pleins), composante

basse fréquence (tirets gris), flux cumulé (trait gras gris). Bas : profils verticaux de courant moyen, concentration moyenne et flux sédimentaire cumulé (premier groupe de vagues en bleu, second en rouge, résiduel en gris clair, total en gris foncé).

ROCHA M.V.L. (2016). Observation and modelling of wave nonlinearities and infragravitywaves in the nearshore. Thèse Université Grenobles-Alpes / Univ. Aveiro. Disponible en ligne sur URL : https://tel.archives-ouvertes.fr/tel-01400356

ROELVINK J.A., STIVE M.J.F. (1989). Bar-generating cross-shore flow mechanisms on a beach. J. Geophys. Res, Vol. 94, pp 4785-4800. https://doi.org/10.1029/JC094iC04p04785 